\title{
Calderón-Zygmund estimates for a class of obstacle problems with nonstandard growth
}

\author{
Jihoon Ok
}

\begin{abstract}
We prove Calderón-Zygmund type estimates for obstacle problems with so-called $L^{p(\cdot)} \log L$-growth. We also find suitable conditions on the variable exponent $p(\cdot)$ and the coefficients of the obstacle problems to obtain desired estimates.
\end{abstract}

Mathematics Subject Classification. Primary 35J92; Secondary 35J20, 35B65.

Keywords. Nonstandard growth, Obstacle problem, Calderón-Zygmund estimate, Variable exponent.

\section{Introduction}

The aim of this paper is to establish optimal higher integrability results for solutions to a class of obstacle problems with non-standard growth conditions. These have been the object of intensive investigation over the last years, after the pioneering papers of Marcellini [36,37]; see for instance also [1$3,7,16,17,25,26,33,34]$. Specifically, we consider obstacle problems involving the following energy functional:

$$
v \in W^{1,1}(\Omega) \mapsto \int_{\Omega} \Phi(x,|D v|) d x, \quad \text { where } \quad \Phi(x, t):=|t|^{p(x)} \log (e+t) .
$$

Here, $\Omega$ is a open subset of $\mathbb{R}^{n}(n \geq 2)$ and $p(\cdot): \Omega \rightarrow \mathbb{R}$ satisfies

$$
1<\gamma_{1} \leq p(\cdot) \leq \gamma_{2}<\infty
$$

for some $\gamma_{1}$ and $\gamma_{2}$. We always assume that $p(\cdot)$ is at least uniformly continuous, hence there exists a modulus of continuity of $p(\cdot)$, say $\omega:[0, \infty) \rightarrow[0, \infty)$, such that $\omega(0)=0$ and it is nondecreasing and concave and satisfies

$$
|p(x)-p(y)| \leq \omega(|x-y|) \quad \text { for all } x, y \in \Omega .
$$

For the function $\Phi: \Omega \times[0, \infty) \rightarrow \mathbb{R}$ given in (1.1), consider the MusielakOrlicz space $L^{\Phi}(\Omega)$ and its Sobolev space $W^{1, \Phi}(\Omega)$, see the next section for 
the definitions of those spaces. Then we fix the function $\psi \in W^{1, \Phi}(\Omega)$, which is called the obstacle, and denote an admissible set by

$$
\mathcal{K}_{\psi}^{\Phi}(\Omega):=\left\{f \in W^{1, \Phi}(\Omega): f \geq \psi \quad \text { a.e. in } \Omega\right\} .
$$

Furthermore, suppose that $F \in L^{\Phi}(\Omega)$ and $\mathbf{a}: \Omega \times \mathbb{R}^{n} \rightarrow \mathbb{R}^{n}$ satisfies

$$
\left\{\begin{array}{l}
|\mathbf{a}(x, \xi)|+\left|D_{\xi} \mathbf{a}(x, \xi)\right||\xi| \leq \Lambda \Phi(x,|\xi|)|\xi|^{-1}=\Lambda|\xi|^{p(x)-1} \log (e+|\xi|) \\
D_{\xi} \mathbf{a}(x, \xi) \eta \cdot \eta \geq \nu \Phi(x,|\xi|)|\xi|^{-2}|\eta|^{2}=\nu|\xi|^{p(x)-2} \log (e+|\xi|)|\eta|^{2}
\end{array}\right.
$$

for all $x \in \Omega, \xi \in \mathbb{R}^{n} \backslash\{0\}$ and $\eta \in \mathbb{R}^{n}$, and for some $0<\nu \leq \Lambda$. Note that the prototype of $\mathbf{a}$ is

$$
\mathbf{a}(x, \xi)=a(x) D_{\xi}[\Phi(x,|\xi|)], \quad \text { where } \quad 0<\nu \leq a(\cdot) \leq \Lambda .
$$

In this setting, we establish a (local) nonlinear Calderón-Zygmund theory for $u \in \mathcal{K}_{\psi}^{\Phi}(\Omega)$ satisfying the variational inequality

$$
\int_{\Omega} \mathbf{a}(x, D u) \cdot D(\varphi-u) d x \geq \int_{\Omega}|F|^{p(x)-2} \log (e+|F|) F \cdot D(\varphi-u) d x
$$

for all $\varphi \in \mathcal{K}_{\psi}^{\Phi}(\Omega)$ with $\varphi-u$ has a compact support in $\Omega$. Precisely, we prove the following implication:

$$
\Phi(\cdot,|D \psi|), \Phi(\cdot,|F|) \in L_{l o c}^{q}(\Omega) \quad \Longrightarrow \quad \Phi(\cdot,|D u|) \in L_{l o c}^{q}(\Omega), \quad q \geq 1 .
$$

under the suitable condition on the variable exponent $p(\cdot)$ and the nonlinearity a, by obtaining corresponding estimates. Note that we will prove the existence and uniqueness of $u \in \mathcal{K}_{\psi}^{\Phi}(\Omega)$ satisfying the variational inequality (1.5) in Appendix, see Sect. 5.

In recent years, concerns about non-autonomous functionals have been rapidly increasing. Those are the functionals

$$
\mathcal{F}(v):=\int_{\Omega} F(x, D v) d x
$$

which have ellipticity or growth properties strongly depending on the space variable $x$. Zhikov have introduced several examples of non-autonomous functionals in the context of Lavrentiev's Phenomenon and the homogenization, see [44]. The one of them is the $p(x)$-Laplace equation for which there have been great research activities in the last two decades. Another example, whose phenomenology makes it strictly connected to the problems we are considering here, is the so-called double phase problem which is described by the functional $\mathcal{F}$ with $F(x, \xi) \approx|\xi|^{p}+a(x)|\xi|^{q}$ or, its borderline case, $\mathcal{F}(x, \xi) \approx$ $|\xi|^{p}+a(x)|\xi|^{p} \log (e+|\xi|)$. Recently, significant regularity results have been obtained by Baroni, Colombo and Mingione in [6,7,16-18]. The functional $F(x, \xi)=\Phi(x,|\xi|)$ we consider in this paper is also an example of nonautonomous functionals. This functional was first considered by Giannetti and Passarelli di Napoli in [27], and then has been studied in [39-41]. The main point of this functional is that it has the properties of both functionals with variable growth and ones with so-called Orlicz growth. Note that if the variable exponent $p(\cdot)$ in $\Phi$ is constant, that is, $p(\cdot) \equiv p$, then $\Phi(x, t)=t^{p} \log (e+t)$ is the typical example of Orlicz functions. The non-autonomous functionals in fact belong to a class of so-called $(p, q)$-growth functionals, which has been first 
introduced by Marcellini in [36,37] and satisfies $|\xi|^{p} \lesssim F(x, \xi) \lesssim|\xi|^{q}$. This has been also under spotlight in recent years, see $[19,33,34]$. We also point out that Harjulehto, Hästö and Klén considered a class of functionals which contains the functionals with $p(\cdot)$-growth, the double phase problems and the functional $\Phi$ in (1.1), see [29].

The nonlinear Calderón-Zygmund theory is the study of the integrability of the gradient of solutions to equations with nonlinear growth, which was first established by Iwaniec for the elliptic $p$-Laplace equations [31]. Since then, DiBenedetto and Manfredi considered the elliptic $p$-Laplace systems [20], and Acerbi and Mingione considered the $p(x)$-Laplace systems and the parabolic $p$-Laplace systems [3,4]. For related to results, we refer to $[5,8,12,13,15,22,32]$ and references therein. Recently, the author of this paper obtained the global version of the implication (1.6) without the term $\Phi(\cdot,|D \psi|)$ for the following equation

$$
\operatorname{div} D_{\xi}[\Phi(x, \xi)]=\operatorname{div}\left(|F|^{p(x)-2} \log (e+|F|) F\right) \quad \text { in } \quad \Omega, \quad u=0 \quad \text { on } \quad \partial \Omega,
$$

under suitable conditions on the variable exponent $p(\cdot)$ and $\Omega$, see [39]. On the other hand, the nonlinear Calderón-Zygmund theory for the obstacle problems was first established by Bögelein et al. [9]. They considered obstacle problems of parabolic $p$-Laplace type. We refer to, for instance, [10,11,23,24,42] for related results for the obstacle problems of $p$-Laplace or $p(x)$-Laplace type.

We point out that the implication (1.6) is not holds for any nonlinearity a satisfying (1.3). In fact, it dose not hold even for any $p(\cdot)$-Laplace equation with a plain continuous function $p(\cdot)$, see [30], or any linear equation with a coefficient matrix which satisfies the uniformly ellipticity condition and the boundedness condition only, see [14]. Therefore, we need extra conditions for $p(\cdot)$ and a. Let us introduce our main assumptions to obtain the implication (1.6).

Assumption Let $\delta>0$ and $R \in(0,1)$.

(AP) $p(\cdot)$ satisfies that

$$
\sup _{0<r \leq R} \omega(r) \log \left(\frac{1}{r}\right) \leq \delta
$$

(AA) a satisfies that

$$
\sup _{0<r \leq R} \sup _{y \in \Omega} f_{B_{r}(y)} \mathcal{O}\left(\mathbf{a}, B_{r}(y)\right)(x) d x \leq \delta,
$$

where

$$
\begin{aligned}
& \mathcal{O}\left(x, B_{r}(y)\right)(x) \\
& \quad:=\sup _{\xi \in \mathbb{R}^{n} \backslash\{0\}}\left|\frac{\mathbf{a}(x, \xi)}{|\xi|^{p(x)-1} \log (e+|\xi|)}-f_{B_{r}(y)} \frac{\mathbf{a}(x, \xi)}{|\xi|^{p(x)-1} \log (e+|\xi|)} d x\right| \leq 2 \Lambda .
\end{aligned}
$$


There are several remarks for the above assumption. In the main result, Theorem 1.1, we will choose sufficiently small $\delta$ which depends on $q$ in (1.6), while $R \in(0,1)$ will be arbitrary given. If $p(\cdot)$ satisfies $(1.8)$, we say $p(\cdot)$ is logHölder continuous in $\Omega$. In the prototype case (1.4), the condition (AA) means that the coefficient function $a(\cdot)$ is a (locally) BMO function with $[a]_{B M O} \leq \delta$. Finally, those assumptions have been naturally arisen in the problems of $p(x)$ Laplace type, see $[10,13]$.

Now we state our main result in this paper. Let

$$
M:=\int_{\Omega}[\Phi(x,|D u|)+\Phi(x,|F|)+\Phi(x,|D \psi|)+1] d x+1 .
$$

Theorem 1.1. Let $\psi \in W^{1, \Phi}(\Omega), F \in L^{\Phi}\left(\Omega, \mathbb{R}^{n}\right)$ and $q \geq 1$. There exists small $\delta=\delta\left(n, \nu, \Lambda, \gamma_{1}, \gamma_{2}, q\right) \in(0,1)$, for which if $p(\cdot)$ and a satisfy (1.8) and (1.9) for some $R \in(0,1)$ and $u \in \mathcal{K}_{\psi}^{\Phi}(\Omega)$ satisfies variational inequality (1.5), then we have the implication (1.6). Moreover, there exists $\eta_{0}=\eta_{0}\left(n, \nu, \Lambda, \gamma_{1}, \gamma_{2}, \omega(\cdot)\right) \in(0,1)$ such that for any $r \in\left(0, \eta_{0} M^{-1}\right)$ and $B_{2 r} \subset \Omega$, we have

$$
\begin{aligned}
f_{B_{r}} \Phi(x,|D u|)^{q} d x \leq & c\left(f_{B_{2 r}} \Phi(x,|D u|) d x\right)^{q} \\
& +c f_{B_{2 r}}\left[\Phi(x,|F|)^{q}+\Phi(x,|D \psi|)^{q}+1\right] d x
\end{aligned}
$$

for some $c=c\left(n, \nu, \Lambda, \gamma_{1}, \gamma_{2}, q\right) \geq 1$.

We would like mention the technique of the proof. As mentioned earlier, in [39] the author proved a similar result for the Eq. (1.7) by using the so-called maximal operator approach due to Caffarelli and Peral [15]. However, in this paper, we prove Theorem 1.1 in a different way introduced by Acerbi and Mingione [4]. Note that the key of this method is the so-called stopping time argument established in [38] first. Moreover, the nonlinearity a considering here not only has the same behavior to $D_{\xi}[\Phi(x,|\xi|)]$ but also contains the coefficient part, see the prototype (1.4), hence we have to consider the effect of coefficient in comparison estimates. At this point, we point out that the structure of a is more complicated than the prototype (1.4). Because, for the prototype (1.4) we can control coefficient $a(\cdot)$ and the variable exponent $p(\cdot)$ independently, while for the nonlinearity a we cannot distinguish the coefficient part and the growth part. This yields more delicate analyses when we derive comparison estimates.

This paper is now organized as follows. Section 1 contains the introduction with main theorem. In Sect. 2, we present notations, basic properties and auxiliary lemmas. In Sect. 3, we derive comparison estimates. In Sect. 4, we prove our main theorem, Theorem 1.1. Finally, in Sect. 5, we prove the existence and uniqueness of the solution to the obstacle problems 


\section{Preliminaries}

\subsection{Notations and properties}

We start with introducing basic notations. For $y \in \mathbb{R}^{n}$ and $r>0, B_{r}(y)$ is a ball in $\mathbb{R}^{n}$ centered $y$ with radius $r$. We shall write $B_{r}=B_{r}(x)$ if the center is not important. For $f \in L_{l o c}^{1}\left(\mathbb{R}^{n}\right)$ and a bounded open set $U$ in $\mathbb{R}^{n},(f)_{U}$ is denoted by the integral average of $f$ in $U$ such that $(f)_{U}=f_{U} f d x=\frac{1}{|U|} \int_{U} f d x$.

We recall $\Phi: \Omega \times[0, \infty) \rightarrow \mathbb{R}$ denoted in (1.1) and, for $1<p<\infty$, define $\Phi: \Omega \times[0, \infty) \rightarrow \mathbb{R}$ by $\Phi_{p}(t):=t^{p} \log (e+t)$. Note that $\Phi(x, t)=\Phi_{p(x)}(t)$. We further denote the conjugate of $\Phi_{p}, \Phi_{p}^{*}:[0, \infty) \rightarrow[0, \infty)$, by

$$
\Phi_{p}^{*}(s):=\sup _{t \geq 0}\left(s t-\Phi_{p}(t)\right) .
$$

We then state elementary properties of $\Phi_{p}$ and $\Phi_{p}^{*}$. we refer to [40] for their proofs.

Proposition 2.1. Let $1<p<\infty, t, s>0$ and $\theta>1$ and $0<\delta<1$.

(1) $\Phi_{p}(\theta t) \leq \theta^{p+1} \Phi_{p}(t)$ and $\Phi_{p}(\delta t) \leq \delta^{p} \Phi_{p}(t)$.

(2) $\Phi_{p}^{*}(\theta s) \leq \theta^{\frac{p}{p-1}} \Phi_{p}^{*}(s)$ and $\Phi_{p}^{*}(\delta s) \leq \delta^{\frac{p+1}{p}} \Phi_{p}^{*}(s)$.

(3) $\Phi_{p}(t+s) \leq \frac{1}{2}\left(\Phi_{p}(2 t)+\Phi_{p}(2 s)\right) \leq 2^{p}\left(\Phi_{p}(t)+\Phi_{p}(s)\right)$.

(4) (Young's inequality) For any $\kappa \in(0,1]$, we have

$$
s t \leq \Phi_{p}\left(\kappa^{\frac{1}{p}} t\right)+\Phi_{p}^{*}\left(\kappa^{-\frac{1}{p}} s\right) \leq \kappa \Phi_{p}(t)+\kappa^{-\frac{1}{p-1}} \Phi_{p}^{*}(s)
$$

and

$$
s t \leq \Phi_{p}^{*}\left(\kappa^{\frac{p-1}{p}} s\right)+\Phi_{p}\left(\kappa^{-\frac{p-1}{p}} t\right) \leq \kappa \Phi_{p}^{*}(s)+\kappa^{-\frac{p^{2}-1}{p}} \Phi_{p}(t) .
$$

(5) There exists $c=c(p)>0$ such that

$$
\Phi_{p}^{*}\left(\Phi_{p}(|\xi|)|\xi|^{-1}\right) \leq c \Phi_{p}(|\xi|) .
$$

We remark that in the above proposition, if $1<\gamma_{1}<p<\gamma_{2}<\infty$, then the constants $c$ depending on $p$ can be replaced by other constants depending on $\gamma_{1}, \gamma_{2}$ instead of $p$.

For $\Phi$ denoted in (1.1) and $N \geq 1$, we denote the Musielak-Orlicz space $L^{\Phi}\left(\Omega, \mathbb{R}^{N}\right)$ by the set of measurable functions $\mathbf{f}: \Omega \rightarrow \mathbb{R}^{N}$ satisfying $\int_{\Omega} \Phi(x,|\mathbf{f}|)$ $d x<\infty$ with the norm

$$
\|\mathbf{f}\|_{L^{\Phi}\left(\Omega, \mathbb{R}^{N}\right)}:=\inf \left\{t>0: \int_{\Omega} \Phi\left(x, \frac{|\mathbf{f}|}{t}\right) d x \leq 1\right\} .
$$

If $N=1$, we simply write $L^{\Phi}(\Omega)=L^{\Phi}\left(\Omega, \mathbb{R}^{1}\right)$. In addition, we denote its Sobolev spaces by

$$
W^{1, \Phi}(\Omega):=\left\{f \in W^{1,1}(\Omega): f,|D f| \in L^{\Phi}(\Omega)\right\} .
$$

with the norm $\|u\|_{W^{1, \Phi}(\Omega)}:=\|u\|_{L^{\Phi}(\Omega)}+\|D u\|_{L^{\Phi}\left(\Omega, \mathbb{R}^{n}\right)}$, and $W_{0}^{1, \Phi}(\Omega)$ by the closure of the set of all functions in $W^{1, \Phi}(\Omega)$ which have compact supports in $\Omega$. We also denote $L^{\Phi^{*}}(\Omega), L^{\Phi_{p}}(\Omega)$ and $W^{1, \Phi_{p}}(\Omega)$ in the same way above by replacing $\Phi$ with $\Phi^{*}$ or $\Phi_{p}$. Note that a Hölder type inequality holds such that

$$
\int_{\Omega} f g d x \leq 2\|f\|_{L^{\Phi}(\Omega)}\|g\|_{L^{\Phi^{*}}(\Omega)},
$$


see [21, Lemma 2.6.5]. Moreover, if $\|f\|_{L^{\Phi}(\Omega)} \geq 1$, one can see that

$$
\|f\|_{L^{\Phi}(\Omega)}^{\gamma_{1}} \leq \int_{\Omega} \Phi(x,|f|) d x .
$$

Indeed, from [21, Lemma 2.1.14], we see that $\|f\|_{L^{\Phi}(\Omega)} \geq 1$ implies $\int_{\Omega} \Phi(x,|f|)$ $d x \geq 1$ and that (2.3) is equivalent to

$$
\int_{\Omega} \Phi\left(x,\left[\int_{\Omega} \Phi(x,|f|) d x\right]^{-\frac{1}{\gamma_{1}}}|f|\right) d x \leq 1
$$

which is proved by the second inequality in (1) of Proposition 2.1 such that

$$
\begin{aligned}
\int_{\Omega} \Phi\left(x,\left[\int_{\Omega} \Phi(x,|f|) d x\right]^{-\frac{1}{\gamma_{1}}}|f|\right) d x & \leq \int_{\Omega}\left[\int_{\Omega} \Phi(x,|f|) d x\right]^{-\frac{p(x)}{\gamma_{1}}} \Phi(x,|f|) d x \\
& \leq \int_{\Omega}\left[\int_{\Omega} \Phi(x,|f|) d x\right]^{-1} \Phi(x,|f|) d x \\
& =1 .
\end{aligned}
$$

Finally, we investigate the monotonicity and the coercivity of the nonlinearity a. From the second condition in (1.3), we have the following monotonicity condition:

$$
\left(\mathbf{a}\left(x, \xi_{1}\right)-\mathbf{a}\left(x, \xi_{2}\right)\right) \cdot\left(\xi_{1}-\xi_{2}\right) \geq \tilde{\nu}\left(\left|\xi_{1}\right|+\left|\xi_{2}\right|\right)^{p(x)-2} \log \left(e+\left|\xi_{1}\right|+\left|\xi_{2}\right|\right)\left|\xi_{1}-\xi_{2}\right|^{2} .
$$

In particular, if $p(x) \geq 2$,

$$
\left(\mathbf{a}\left(x, \xi_{1}\right)-\mathbf{a}\left(x, \xi_{2}\right)\right) \cdot\left(\xi_{1}-\xi_{2}\right) \geq \tilde{\nu} \Phi\left(x,\left|\xi_{1}-\xi_{2}\right|\right) .
$$

Moreover, letting $\xi_{1}=\xi$ and $\xi_{2}=0$ in (2.4), we have

$$
\mathbf{a}(x, \xi) \cdot \xi \geq \tilde{\nu} \Phi(x,|\xi|) \text {. }
$$

\subsection{Auxiliary lemmas}

We first state the local self improving property to the gradient of weak solutions to the following homogeneous equation

$$
\operatorname{div} \mathbf{a}(x, D w)=0 \quad \text { in } \quad B_{2 r}
$$

The proof is almost same to, rather simpler than, the one of [39, Theorem 3.7]. Therefore, we omit to prove the next lemma here.

Lemma 2.2. Let $M_{1} \geq 1$. Suppose that $\mathbf{a}: \Omega \times \mathbb{R}^{n} \rightarrow \mathbb{R}^{n}$ satisfies (1.3) and $p(\cdot): \Omega \rightarrow \mathbb{R}$ does (1.2) and (1.8) for some $\delta>0$ and $R \in(0,1)$. Then there exist $\sigma_{0}=\sigma_{0}\left(n, \nu, \Lambda, \gamma_{1}, \gamma_{2}, \delta\right) \in(0,1)$ and $\eta_{1}=\eta_{1}\left(n, \gamma_{1}, \gamma_{2}, \omega(\cdot)\right) \in(0,1)$ such that for any $B_{2 r} \subset \subset \Omega$ with $r \leq \frac{\eta_{1} R}{M_{1}}$, if $w \in W^{1, \Phi}\left(B_{2 r}\right)$ is a weak solution to (2.7) with

$$
\int_{B_{2 r}}[\Phi(x,|D w|)+1] d x+1 \leq M_{1},
$$

then $\Phi(x,|D w|) \in L^{1+\sigma_{0}}\left(B_{r}\right)$ with the following estimates: for any $0<\sigma \leq \sigma_{0}$,

$$
f_{B_{r}} \Phi(x,|D w|)^{1+\sigma} d x \leq c\left\{\left(f_{B_{2 r}} \Phi(x,|D w|) d x\right)^{1+\sigma}+1\right\}
$$


for some $c=c\left(n, \nu, \Lambda, \gamma_{1}, \gamma_{2}, \delta\right) \geq 1$. Moreover, if $\delta \in(0,1)$, one can find $\sigma_{0}$ and $c$ independent of $\delta$.

Without loss of generality, we always assume that

$$
\sigma_{0} \leq 4\left(\gamma_{1}-1\right)
$$

Remark 2.3. The previous lemma still holds true under milder conditions on the nonlinearity a. Precisely, even if a only satisfies that

$$
|\mathbf{a}(x, \xi)| \leq \Lambda|\xi|^{p(x)-1} \log (e+|\xi|),
$$

instead of (1.3), and (2.6), we can obtain the same result in the previous lemma.

The next lemma gives the gradient bound of weak solutions to homogenous equations related to the function $\Phi_{p}$, which is the special case of the result in [35, Lemma 5.1].

Lemma 2.4. Let $1<p<\infty$. Suppose $v \in W^{1, \Phi_{p}}\left(B_{2 r}\right)$ be a weak solution to

$$
\operatorname{div} \overline{\mathbf{b}}(D v)=0 \quad \text { in } \quad B_{2 r},
$$

where $\overline{\mathbf{b}}: \mathbb{R}^{n} \rightarrow \mathbb{R}^{n}$ satisfies

$$
\left\{\begin{array}{l}
|\overline{\mathbf{b}}(\xi)|+\left|D_{\xi} \overline{\mathbf{b}}(\xi)\right||\xi| \leq \Lambda \Phi_{p}(|\xi|)|\xi|^{-1}=\Lambda|\xi|^{p-1} \log (e+|\xi|) \\
D_{\xi} \overline{\mathbf{b}}(\xi) \eta \cdot \eta \geq \nu \Phi_{p}(|\xi|)|\xi|^{-2}|\eta|^{2}=\nu|\xi|^{p_{2}-2} \log (e+|\xi|)|\eta|^{2}
\end{array}\right.
$$

for all $\xi \in \mathbb{R}^{n} \backslash\{0\}$ and $\eta \in \mathbb{R}^{n}$. Then we have

$$
\sup _{B_{r}} \Phi_{p}(|D v|) \leq c f_{B_{2 r}} \Phi_{p}(|D v|) d x
$$

for some $c=c(n, \nu, \Lambda, p)>0$.

We end this section introducing a technical lemma, see [28, Lemma 4.3].

Lemma 2.5. Let $\phi$ be a bounded nonnegative function on $\left[r_{1}, r_{2}\right]$. Suppose that for any $s_{1}, s_{2}$ with $0<r_{1} \leq s_{1}<s_{2} \leq r_{2}$,

$$
\phi\left(s_{1}\right) \leq \eta \phi\left(s_{2}\right)+\frac{C_{1}}{\left(s_{2}-s_{1}\right)^{\theta}}+C_{2},
$$

where $\theta, C_{1}, C_{2} \geq 0$ and $\eta \in(0,1)$. Then for any $r_{1} \leq s_{1}<s_{2} \leq r_{2}$, it holds that

$$
\phi\left(r_{1}\right) \leq c\left[\frac{C_{1}}{\left(s_{2}-s_{1}\right)^{\theta}}+C_{2}\right]
$$

for some $c=c(\eta, \theta)>0$. 


\section{Comparison estimates}

In this section, we shall obtain two comparison estimates, which will be obtained by comparing between the variational inequality (1.5) and homogeneous equations related to the functions $\Phi$ and $\Phi_{p}$, respectively, see (3.2) and (3.14). Note that, by Lemma 2.4, weak solutions to (3.14) have the bounded gradients.

Let us start with a comparison principle.

Lemma 3.1. Let $\psi, w \in W^{1, \Phi}(U), U \subset \Omega$, satisfy

$$
\left\{\begin{array}{l}
-\operatorname{div} \mathbf{a}(x, D \psi) \leq-\operatorname{div} \mathbf{a}(x, D w) \text { in } U \\
\psi \leq w \text { on } \partial U
\end{array}\right.
$$

in the weak sense, that is, $(\psi-w)^{+} \in W_{0}^{1, \Phi}(\Omega)$ and

$$
\int_{U}(\mathbf{a}(x, D \psi)-\mathbf{a}(x, D w)) \cdot D \varphi d x \leq 0
$$

for all $\varphi \in W_{0}^{1, \Phi}(U)$ with $\varphi \geq 0$. Then we have $\psi \leq w$ a.e. in $U$.

Proof. By taking $\varphi=(\psi-w)_{+}$in the above inequality and using (2.4), we have

$\int_{\{x \in U: \psi(x)>w(x)\}}(|D \psi|+|D w|)^{p(x)} \log (e+|D \psi|+|D w|)|D \psi-D w|^{2} d x \leq 0$,

which implies that $\psi \leq w$ a.e. in $U$.

Let $u \in \mathcal{K}_{\psi}^{\Phi}(\Omega)$ satisfy the variational inequality to (1.5) and $r>0$ be a sufficiently small number to be determined later, see (3.8) and (3.12). Fix $B_{4 r} \Subset \Omega$. We then consider two comparison maps;

$$
\begin{gathered}
\left\{\begin{array}{l}
-\operatorname{div} \mathbf{a}(x, D k)=-\operatorname{div} \mathbf{a}(x, D \psi) \text { in } B_{4 r}, \\
k=u \text { on } \partial B_{4 r},
\end{array}\right. \\
\left\{\begin{array}{l}
-\operatorname{div} \mathbf{a}(x, D w)=0 \text { in } B_{4 r}, \\
w=k \text { on } \partial B_{4 r} .
\end{array}\right.
\end{gathered}
$$

Note that the existence and the uniqueness of weak solutions to the above equations can be proved in a similar way in the proof of Theorem 5.1.

Lemma 3.2. Let $k$ and $w$ in $W^{1, \Phi}\left(B_{4 r}\right)$ be the weak solutions to (3.1) and (3.2), respectively. Then we have

$$
\begin{aligned}
\int_{B_{4 r}} \Phi(x,|D k|) d x & \leq c \int_{B_{4 r}}[\Phi(x,|D u|)+\Phi(x,|D \psi|)] d x \\
\int_{B_{4 r}} \Phi(x,|D w|) d x & \leq c \int_{B_{4 r}} \Phi(x,|D k|) d x \\
& \leq c \int_{B_{4 r}}[\Phi(x,|D u|)+\Phi(x,|D \psi|)] d x
\end{aligned}
$$

for any $\kappa \in(0,1)$, 


$$
\begin{aligned}
\int_{B_{4 r}} \Phi(x,|D u-D w|) d x \leq & \kappa f_{B_{4 r}} \Phi(x,|D u|) d x \\
& +c(\kappa) f_{B_{4 r}}[\Phi(x,|F|)+\Phi(x, D \psi)] d x
\end{aligned}
$$

for some $c=c\left(n, \nu, \Lambda, \gamma_{1}, \gamma_{2}\right)>0$ and $c(\kappa)=c\left(n, \nu, \Lambda, \gamma_{1}, \gamma_{2}, \kappa\right) \geq 1$.

Proof. By taking $k-u$ as a test function in the weak formulation of (3.1), and using (2.6), (1.3) and (2.1), we have

$$
\begin{aligned}
\int_{B_{4 r}} \Phi(x, D k) d x & \leq c \int_{B_{4 r}} \mathbf{a}(x, D k) \cdot D k d x \\
& =c \int_{B_{4 r}}[\mathbf{a}(x, D k) \cdot D u+\mathbf{a}(x, D \psi) \cdot(D k-D u)] d x \\
& \leq \frac{1}{2} \int_{B_{4 r}} \Phi(x, D k) d x+c \int_{B_{4 r}}[\Phi(x, D u)+\Phi(x, D \psi)] d x,
\end{aligned}
$$

which implies (3.3). Similarly, by taking $w-k$ as a test function in the weak formulation of (3.2) and using (3.3), we obtain (3.4).

It remains to show (3.5). Since $\psi \leq k$ a.e. in $B_{4 r}$ by Lemma 3.1 and $u=k$ on $\partial B_{4 r}$, extending $k$ by $u$ in $\Omega \backslash B_{4 r}$, we have $k \in \mathcal{K}_{\psi}^{\Phi}(\Omega)$. Therefore by taking $k$ and $u-k$ as test functions on (1.5) and (3.1), respectively, and using (2.4) and (2.1), we have

$$
\begin{aligned}
& \int_{B_{4 r}}(|D u|+|D k|)^{p(x)-2} \log (e+|D u|+|D k|)|D u-D k|^{2} d x \\
& \leq \frac{1}{\tilde{\nu}} \int_{B_{4 r}}(\mathbf{a}(x, D u)-\mathbf{a}(x, D k)) \cdot(D u-D k) d x \\
& \leq \frac{1}{\tilde{\nu}} \int_{B_{4 r}}\left(|F|^{p(x)-2} \log (e+|F|) F+\mathbf{a}(x, D \psi)\right) \cdot(D u-D k) d x \\
& \leq c \int_{B_{4 r}}\left(\Phi(x,|F|)|F|^{-1}+\Phi(x,|D \psi|)|D \psi|^{-1}\right) \cdot(D u-D k) d x \\
& \leq \frac{1}{2} \int_{B_{4 r}} \Phi(x,|D u-D k|) d x+c \int_{B_{4 r}}[\Phi(x,|F|)+\Phi(x,|D \psi|)] d x .
\end{aligned}
$$

At this point, if $p(x)<2$, we have from Young's inequality that for any $\kappa \in(0,1)$,

$$
\begin{aligned}
\Phi(x,|D u-D k|)= & (|D u|+|D k|)^{\frac{(2-p(x)) p(x)}{2}} \\
& \times(|D u|+|D k|)^{\frac{(p(x)-2) p(x)}{2}}|D u-D k|^{p(x)} \log (e+|D u-D k|) \\
\leq & \kappa 2^{\gamma_{2}}(1+\log 2)(\Phi(x,|D u|)+\Phi(x,|D k|)) \\
& +c(\kappa)(|D u|+|D k|)^{p(x)-2} \log (e+|D u|+|D k|)|D u-D k|^{2}
\end{aligned}
$$


for some $c(\kappa)=c\left(n, \gamma_{1}, \gamma_{2}, \kappa\right) \geq 1$. Note by (2.5) that (3.6) also holds for $p(x) \geq 2$. Therefore, combining two previous inequalities and using (3.3), we have for any $\kappa \in(0,1)$,

$$
\begin{aligned}
\int_{B_{4 r}} \Phi(x,|D u-D k|) d x \leq & \frac{\kappa}{2} \Phi(x,|D u|) d x+c(\kappa) \int_{B_{4 r}}[\Phi(x,|F|) \\
& +\Phi(x,|D \psi|)] d x .
\end{aligned}
$$

In a similar way above, by testing $k-w$ in (3.1) and (3.2), we have

$$
\int_{B_{4 r}} \Phi(x,|D k-D w|) d x \leq \frac{\kappa}{2} \int_{B_{4 r}} \Phi(x,|D u|) d x+c(\kappa) \int_{B_{4 r}} \Phi(x,|D \psi|) d x .
$$

Therefore, we obtain (3.5).

Note that from (3.3) and (3.4),

$$
\int_{B_{4 r}}[\Phi(x,|D w|)+1] d x+1 \leq c_{0} M
$$

for some $c_{0}=c_{0}\left(n, \nu, \Lambda, \gamma_{1}, \gamma_{2}\right) \geq 1$, where $M$ is denoted in (1.11).

Suppose $r>0$ satisfies that

$$
r \leq \frac{\eta_{1} R}{2 c_{0} M} \quad \text { and } \quad \omega(4 r) \leq \min \left\{\frac{\sigma_{0}}{4}, 1\right\},
$$

where $\sigma_{0}, \eta_{1} \in(0,1)$ are determined in Lemma 2.2, and set

$$
p_{2}:=\sup _{B_{2 r}} p(\cdot) \quad \text { and } \quad p_{1}:=\inf _{B_{2 r}} p(\cdot) .
$$

Then, in view of Lemma 2.2 with $\left(r, M_{1}\right)$ replaced by $\left(2 r, c_{0} M\right)$, we have $\Phi(x,|D w|) \in L^{1+\sigma_{0}}\left(B_{2 r}\right)$ with the estimates

$$
f_{B_{2 r}} \Phi(x,|D w|)^{1+\sigma} d x \leq c\left(f_{B_{4 r}} \Phi(x,|D w|) d x\right)^{1+\sigma}+c .
$$

for all $\sigma \in\left(0, \sigma_{0}\right]$. In particular, since $p_{2}-p_{1} \leq \omega(4 r) \leq \sigma_{0} / 4$ by (3.8) and (2.8), using the previous inequality when $\sigma=\omega(4 r)$ and (3.4) we have

$$
\begin{aligned}
\int_{B_{2 r}} \Phi_{p_{2}}(|D w|) d x & \leq c\left\{\left(f_{B_{4 r}} \Phi(x,|D w|) d x\right)^{1+\omega(4 r)}+1\right\} \\
& \leq c\left(f_{B_{4 r}} \Phi(x,|D w|) d x+1\right) \\
& \leq c\left(f_{B_{4 r}}[\Phi(x,|D u|)+\Phi(x,|D \psi|)] d x+1\right) .
\end{aligned}
$$

In the last estimate above we have used the fact from (3.7), (3.8) and (1.8) that

$$
\left(f_{B_{4 r}} \Phi(x,|D w|) d x\right)^{\omega(4 r)} \leq c(4 r)^{-(n+1) \omega(4 r)} \leq c e^{(n+1) \delta} \leq c .
$$


Similarly, since $p_{2}\left(1+\sigma_{0} / 4\right) \leq p(x)\left(1+\sigma_{0} / 4\right)+\omega(4 r)\left(1+\sigma_{0} / 4\right) \leq p(x)(1+$ $\sigma+\omega(4 r))$, we have

$$
\begin{aligned}
& f_{B_{2 r}} \Phi_{p_{2}}(|D w|)^{1+\sigma_{0} / 4} d x \\
& \leq c\left\{\left(f_{B_{4 r}} \Phi(x,|D w|) d x\right)^{1+\sigma_{0} / 4+\omega(4 r)}+1\right\} \\
& \leq c\left\{\left(f_{B_{4 r}}[\Phi(x,|D u|)+\Phi(x,|D \psi|)] d x\right)^{1+\sigma_{0} / 4}+1\right\} .
\end{aligned}
$$

We will construct another comparison map. Consider $\mathbf{b}: B_{2 r} \times \mathbb{R}^{n}$ denoted by

$$
\mathbf{b}(x, \xi):=|\xi|^{p_{2}-p(x)} \mathbf{a}(x, \xi)
$$

Then, we have

$$
D_{\xi} \mathbf{b}(x, \xi)=\left(p_{2}-p(x)\right)|\xi|^{p_{2}-p(x)-2} \xi \otimes \mathbf{a}(x, \xi)+|\xi|^{p_{2}-p(x)} D_{\xi} \mathbf{a}(x, \xi),
$$

hence by (1.3) it follows that

$$
\left\{\begin{array}{l}
|\mathbf{b}(x, \xi)|+\left|D_{\xi} \mathbf{b}(x, \xi)\right||\xi| \leq 3 \Lambda \Phi_{p_{2}}(|\xi|)|\xi|^{-1}=3 \Lambda|\xi|^{p_{2}-1} \log (e+|\xi|) \\
D_{\xi} \mathbf{b}(x, \xi) \eta \cdot \eta \geq \frac{\nu}{2} \Phi_{p_{2}}(|\xi|)|\xi|^{-2}|\eta|^{2}=\frac{\nu}{2}|\xi|^{p_{2}-2} \log (e+|\xi|)|\eta|^{2}
\end{array}\right.
$$

for all $x \in \Omega, \xi \in \mathbb{R}^{n} \backslash\{0\}$ and $\eta \in \mathbb{R}^{n}$, provided that

$$
\omega(4 r) \leq \min \left\{1, \frac{\nu}{2 n^{2}}\right\} .
$$

Further we denote $\overline{\mathbf{b}}: \mathbb{R}^{n} \rightarrow \mathbb{R}^{n}$ by

$$
\overline{\mathbf{b}}(\xi):=\int_{B_{2 r}} \mathbf{b}(x, \xi) d x
$$

Then we see that (3.11) with $\mathbf{b}$ replaced by $\overline{\mathbf{b}}$ also holds and, by (1.10),

$$
|\mathbf{b}(x, \xi)-\overline{\mathbf{b}}(\xi)| \leq \mathcal{O}\left(\mathbf{a}, B_{2 r}\right)(x)|\xi|^{p_{2}-1} \log (e+|\xi|)
$$

for all $x \in B_{2 r}$ and $\xi \in \mathbb{R}^{n}$. Now we consider the following equation:

$$
\left\{\begin{array}{l}
-\operatorname{div} \overline{\mathbf{b}}(D v)=0 \text { in } B_{2 r}, \\
v=w \text { on } \partial B_{2 r} .
\end{array}\right.
$$

Then, making use of the same method estimating (3.3) along with (3.9) and (3.4), we have

$$
\begin{aligned}
\int_{B_{2 r}} \Phi_{p_{2}}(|D v|) d x & \leq c \int_{B_{2 r}} \Phi_{p_{2}}(|D w|) d x \\
& \leq c\left(f_{B_{4 r}}[\Phi(x,|D u|)+\Phi(x,|D \psi|)] d x+1\right) .
\end{aligned}
$$

In addition, by Lemma 2.4 , 


$$
\begin{aligned}
\sup _{B_{r}} \Phi_{p_{2}}(|D v|) & \leq c \int_{B_{2 r}} \Phi_{p_{2}}(|D v|) d x \\
& \leq c\left(\int_{B_{4 r}}[\Phi(x,|D u|)+\Phi(x,|D \psi|)] d x+1\right)
\end{aligned}
$$

for some $c=c\left(n, \nu, \Lambda, \gamma_{1}, \gamma_{2}\right)>0$.

Finally, we derive the comparison estimate between $w$ and $v$.

Lemma 3.3. Let $w \in W^{1, \Phi}\left(B_{4 r}\right) \cap W^{1, \Phi_{p_{2}}}\left(B_{2 r}\right)$ and $v \in W^{1, \Phi_{p_{2}}}\left(B_{4 r}\right)$ be weak solutions to (3.2) and (3.14), respectively, where $r$ satisfies (3.8) and (3.12). Then we have for any $\kappa \in(0,1)$,

$$
\begin{aligned}
& f_{B_{2 r}} \Phi_{p_{2}}(|D w-D v|) d x \\
& \quad \leq\left(\kappa+c(\kappa) \delta^{\frac{\sigma_{0} / 4}{1+\sigma_{0} / 4}}\right)\left(f_{B_{4 r}}[\Phi(x,|D u|)+\Phi(x,|D \psi|)] d x+1\right)
\end{aligned}
$$

for some $c(\kappa) \geq 1$ depending on $n, \nu, \Lambda, \gamma_{1}, \gamma_{2}, \kappa$.

Proof. By testing $w-v \in W_{0}^{1, \Phi_{p_{2}}}\left(B_{2 r}\right) \subset W_{0}^{1, \Phi}\left(B_{2 r}\right)$, we have

$$
\begin{aligned}
I_{1}:= & f_{B_{2 r}}(\overline{\mathbf{b}}(D w)-\overline{\mathbf{b}}(D v)) \cdot(D w-D v) d x \\
\leq & f_{B_{2 r}}(\mathbf{b}(x, D w)-\mathbf{a}(x, D w)) \cdot(D w-D v) d x \\
& +f_{B_{2 r}}(\overline{\mathbf{b}}(D w)-\mathbf{b}(x, D w)) \cdot(D w-D v) d x=: I_{2}+I_{3} .
\end{aligned}
$$

Using a similar technique estimating (3.6) along with (3.15), (3.9) and (3.4), we have for any $\kappa \in(0,1)$,

$$
f_{B_{2 r}} \Phi_{p_{2}}(|D w-D v|) d x \leq \frac{\kappa}{2}\left(f_{B_{4 r}}[\Phi(x,|D u|)+\Phi(x,|D \psi|)] d x\right)+c_{1}(\kappa) I_{1}
$$

for some $c_{1}(\kappa)=c_{1}\left(n, \nu, \Lambda, \gamma_{1}, \gamma_{2}, \kappa\right) \geq 1$. We next estimate $I_{2}$. Note that applying the mean value theorem to the map $t \in[0,1] \mapsto|\xi|^{t\left(p_{2}-p(x)\right)}$ we have

$$
|\xi|^{p_{2}-p(x)}-1=\left(p_{2}-p(x)\right)|\xi|^{t_{\xi}\left(p_{2}-p(x)\right)} \log |\xi| \quad \text { for some } t_{\xi} \in(0,1),
$$

which implies

$|\mathbf{b}(x, D w)-\mathbf{a}(x, D w)| \leq c w(4 r)|D w|^{t_{D w}\left(p_{2}-p(x)\right)+p(x)-1} \log (e+|D w|) \log |D w|$.

Since the previous quantity is bounded when $|D w| \leq 1$, we obtain

$$
|\mathbf{b}(x, D w)-\mathbf{a}(x, D w)| \leq c w(4 r)\left(|D w|^{p_{2}-1} \log ^{2}(e+|D w|)+1\right),
$$

and so

$$
\left|I_{2}\right| \leq c \omega(4 r) f_{B_{2 r}}\left(|D w|^{p_{2}-1} \log ^{2}(e+|D w|)+1\right)|D w-D v| d x .
$$


At this stage, we follows the same computations in the proof of [39, Lemma 4.3], see p.25 - p.26 in there. Then we obtain

$$
\left|I_{2}\right| \leq c \omega(4 r) \log \left(\frac{1}{4 r}\right)\left(f_{B_{4 r}}\left[\Phi_{p_{2}}(|D w|)+\Phi_{p_{2}}(|D \psi|)\right] d x+1\right) .
$$

Finally, we estimate $I_{3}$. By (3.13), (2.1), (2.2), (1.10) and Hölder's inequality, we have for any $\tilde{\kappa} \in(0,1)$

$$
\begin{aligned}
\left|I_{3}\right| \leq & \int_{B_{2 r}} \mathcal{O}\left(\mathbf{a}, B_{2 r}\right)|D w|^{p_{2}-1} \log (e+|D w|)|D w-D v| d x \\
\leq & \int_{B_{2 r}} \mathcal{O}\left(\mathbf{a}, B_{2 r}\right)\left[c(\tilde{\kappa}) \Phi_{p_{2}}(|D w|)+\frac{\tilde{\kappa}}{2 \Lambda} \Phi_{p_{2}}(|D w-D v|)\right] d x \\
\leq & \tilde{\kappa} \int_{B_{2 r}} \Phi_{p_{2}}(|D w-D v|) d x \\
& +c(\tilde{\kappa})\left(f_{B_{2 r}} \mathcal{O}\left(\mathbf{a}, B_{2 r}\right) d x\right)^{\frac{\sigma_{0} / 4}{1+\sigma_{0} / 4}}\left(\int_{B_{2 r}} \Phi_{p_{2}}(|D w|)^{1+\sigma_{0} / 4} d x\right)^{\frac{1}{1+\sigma_{0} / 4}} .
\end{aligned}
$$

Consequently, Combining (3.18)-(3.20), choosing $\tilde{\kappa}=\frac{1}{2 c_{1}(\kappa)}$ and using (3.9), $(3.10),(3.15)$ and the definition of the $(\delta, R)$-vanishing condition for $p(\cdot)$ and a, we obtain (3.17).

\section{Proof of Theorem 1.1}

In this section, we prove Theorem 1.1. In fact, it suffices to show the estimate (1.12). $R \in(0,1)$ be an arbitrary given number, while $\delta>0$ will be determined later. With this $R, \delta$, we assume that $p(\cdot)$ and a satisfies (1.8) and (1.9), respectively.

Let $r>0$ satisfy that

$$
r \leq \frac{2 \eta_{1} R}{c_{0} M} \quad \text { and } \quad \omega(r) \leq \min \left\{\frac{\sigma_{0}}{4}, \frac{\nu}{2 n^{2}}, 1\right\}
$$

and fix $B_{2 r} \Subset \Omega$. Define

$$
\lambda_{0}:=f_{B_{2 r}}\left[\Phi(x,|D u|)+\frac{1}{\delta}\{\Phi(x,|F|)+\Phi(x,|D \psi|)\}\right] d x+1
$$

and, for $1 \leq s \leq 2$ and $\lambda>0$, the supper-level set of $\Phi(\cdot,|D u(\cdot)|)$ by

$$
E(s, \lambda):=\left\{x \in B_{s r}: \Phi(x,|D u(x)|)>\lambda\right\} .
$$

Lemma 4.1. Fix any $1 \leq s_{1}<s_{2} \leq 2$. If

$$
\lambda>A_{1} \lambda_{0} \geq 1, \quad \text { where } A_{1}:=\left(\frac{40}{s_{2}-s_{1}}\right)^{n} .
$$


Then there exist mutually disjoint balls $B_{r_{i}}\left(x_{i}\right), i=1,2,3, \ldots$, where $x_{i} \in$ $E\left(s_{1}, \lambda\right)$ and $r_{i} \in\left(0, \frac{\left(s_{2}-s_{1}\right) r}{20}\right]$, such that for each $i$

$$
f_{B_{r_{i}}\left(x_{i}\right)}\left[\Phi(x,|D u|)+\frac{1}{\delta}\{\Phi(x,|F|)+\Phi(x,|D \psi|)\}\right] d x=\lambda,
$$

for any $\rho \in\left(r_{i},\left(s_{2}-s_{1}\right) r\right)$,

$$
f_{B_{\rho}\left(x_{i}\right)}\left[\Phi(x,|D u|)+\frac{1}{\delta}\{\Phi(x,|F|)+\Phi(x,|D \psi|)\}\right] d x<\lambda,
$$

and

$$
E\left(s_{1}, \lambda\right) \backslash N \subset \bigcup_{i=1}^{\infty} B_{5 r_{i}}\left(x_{i}\right)
$$

for some a measure zero set $N$.

Proof. For $\rho>0$ and $x \in E\left(s_{1}, \lambda\right)$, let us set

$$
I(x, \rho):=f_{B_{\rho}(x)}\left[\Phi(x,|D u|)+\frac{1}{\delta}\{\Phi(x,|F|)+\Phi(x,|D \psi|)\}\right] d x .
$$

Then, by (4.2),

$$
I(x, \rho) \leq\left(\frac{40}{s_{2}-s_{1}}\right)^{n} \lambda_{0}<\lambda \quad \text { for all } \rho \in\left(\frac{\left(s_{2}-s_{1}\right) r}{20},\left(s_{2}-s_{1}\right) r\right) .
$$

On the other hand, by the definition of $E\left(s_{1}, \lambda\right)$ and Lebesgue's differentiation theorem, we have

$$
\lim _{\rho \rightarrow 0} I(x, \rho)>\lambda \quad \text { for a.e. } x \in E\left(s_{1}, \lambda\right) .
$$

Since $I$ is continuous for $\rho$, for a.e. $x \in E\left(s_{1}, \lambda\right)$ there exists $r_{x} \in\left(0, \frac{\left(s_{2}-s_{1}\right) r}{20}\right)$ such that

$$
I\left(x, r_{x}\right)=\lambda \quad \text { and } \quad I(x, \rho)<\lambda \quad \text { for all } \rho \in\left(r_{x},\left(s_{2}-s_{1}\right) r\right) .
$$

Therefore, by Vitali's covering lemma, we can obtain the desired result.

Under the results of the above lemma, setting

$$
B^{i}=B_{5 r_{i}}\left(x_{i}\right), \quad j B^{i}:=B_{5 j r_{i}}\left(x_{i}\right)(j=2,3,4), \quad p_{i 2}:=\sup _{2 B^{i}} p(\cdot) .
$$

we have from (4.5) that

$$
f_{4 B^{i}} \Phi(x,|D u|) d x \leq \lambda \text { and } f_{4 B^{i}}[\Phi(x,|F|)+\Phi(x,|D \psi|)] d x \leq \delta \lambda .
$$

Then, in view of Lemma 3.2, Lemma 3.3 and Lemma (3.16), we have the following result. Note that from (4.1) and the fact that $5 r_{i} \leq r / 4$, the all restrictions of $r>0$ in the previous section, see (3.8) and (3.12), are satisfied when $r=5 r_{i}$. 
Lemma 4.2. For any $\epsilon \in(0,1)$ there exists small $\delta\left(n, \nu, \Lambda, \gamma_{1}, \gamma_{2}, \epsilon\right) \in(0,1)$ such that the following holds: under the above setting, for each $i=1,2,3, \ldots$, there exist $w_{i} \in W^{1, \Phi}\left(4 B^{i}\right) \cap W^{1, \Phi_{p_{i 2}}}\left(2 B^{i}\right)$ and $v_{i} \in W^{1, \Phi_{p_{i 2}}}\left(2 B^{i}\right) \cap W^{1, \infty}\left(B^{i}\right)$ such that

$$
f_{B^{i}} \Phi\left(x,\left|D u-D w_{i}\right|\right) d x+\int_{B^{i}} \Phi_{p_{i 2}}\left(\left|D w_{i}-D v_{i}\right|\right) d x \leq \epsilon \lambda
$$

and

$$
\sup _{x \in B^{i}} \Phi\left(x,\left|D v_{i}\right|\right) \leq c_{L} \lambda
$$

for some $c_{L}=c_{L}\left(n, \nu, \Lambda, \gamma_{1}, \gamma_{2}\right)>0$.

Proof of the estimate (1.12). We first estimate

$$
\int_{E\left(s_{1}, A_{2} \lambda\right)} \Phi(x,|D u|) d x, \quad \text { where } A_{2}:=2 \cdot 4^{\gamma_{2}}\left(c_{L}+2\right) .
$$

From (4.6) we see $E\left(s_{1}, \lambda\right) \backslash N \subset \bigcup_{i=1}^{\infty} B^{i}$, hence

$$
\int_{E\left(s_{1}, A_{2} \lambda\right)} \Phi(x,|D u|) d x \leq \sum_{i=1}^{\infty} \int_{E\left(s_{1}, A_{2} \lambda\right) \cap B^{i}} \Phi(x,|D u|) d x .
$$

By (3) of Proposition 2.1 and (4.8), we have for $x \in E\left(s_{1}, A_{2} \lambda\right) \cap B^{i}$

$$
\begin{aligned}
\Phi(x,|D u|) & \leq 4^{\gamma_{2}}\left(\Phi\left(x,\left|D u-D w_{i}\right|\right)+\Phi_{p_{i 2}}\left(\left|D w_{i}-D v_{i}\right|\right)+\Phi_{p_{i 2}}\left(\left|D v_{i}\right|\right)+2\right) \\
& \leq 4^{\gamma_{2}}\left(\Phi\left(x,\left|D u-D w_{i}\right|\right)+\Phi_{p_{i 2}}\left(\left|D w_{i}-D v_{i}\right|\right)+\left(c_{L}+2\right) \lambda\right) \\
& \leq 4^{\gamma_{2}}\left(\Phi\left(x,\left|D u-D w_{i}\right|\right)+\Phi_{p_{i 2}}\left(\left|D w_{i}-D v_{i}\right|\right)\right)+\frac{1}{2} \Phi(x,|D u|),
\end{aligned}
$$

which together with (4.7) imply

$$
\int_{E\left(s_{1}, A_{2} \lambda\right)} \Phi(x,|D u|) d x \leq 2 \cdot 4^{\gamma_{2}} \epsilon \lambda \sum_{i=1}^{\infty}\left|B^{i}\right|=2 \cdot 4^{\gamma_{2}} 5^{n} \epsilon \lambda \sum_{i=1}^{\infty}\left|B_{r_{i}}\left(x_{i}\right)\right| .
$$

Note that by (4.4) we have,

$$
\frac{\lambda}{2} \leq f_{B_{r_{i}}\left(x_{i}\right)} \Phi(x,|D u|) d x \quad \text { or } \quad \frac{\delta \lambda}{2} \leq f_{B_{r_{i}}\left(x_{i}\right)}[\Phi(x,|F|)+\Phi(x,|D \psi|)] d x .
$$

Then, the first case yields

$$
\left|B_{r_{i}}\left(x_{i}\right)\right| \leq \frac{4}{\lambda} \int_{\left\{x \in B_{r_{i}}\left(x_{i}\right): \Phi(x,|D u|)>\frac{\lambda}{4}\right\}} \Phi(x,|D u|) d x,
$$

and the second case does

$$
\left|B_{r_{i}}\left(x_{i}\right)\right| \leq \frac{4}{\delta \lambda} \int_{\left\{x \in B_{r_{i}}\left(x_{i}\right): \Phi(x,|F|)+\Phi(x,|D u|)>\frac{\delta \lambda}{4}\right\}}[\Phi(x,|F|)+\Phi(x,|D \psi|)] d x .
$$

Inserting these two estimates into (4.10) and using the fact that $B_{r_{i}}\left(x_{i}\right), i=$ $1,2, \ldots$, are mutually disjoint and contained in $B_{s_{2} r}$, we finally obtain 


$$
\begin{aligned}
\int_{E\left(s_{1}, A_{2} \lambda\right)} & \Phi(x,|D u|) d x \leq c_{1} \epsilon\left\{\int_{\left\{x \in B_{s_{2} r}: \Phi(x,|D u|)>\frac{\lambda}{4}\right\}} \Phi(x,|D u|) d x\right. \\
& \left.+\frac{1}{\delta} \int_{\left\{x \in B_{s_{2} r}: \Phi(x,|F|)+\Phi(x,|D u|)>\frac{\delta \lambda}{4}\right\}}[\Phi(x,|F|)+\Phi(x,|D \psi|)] d x\right\}
\end{aligned}
$$

for some $c_{1}=c_{1}\left(n, \nu, \Lambda, \gamma_{1}, \gamma_{2}\right) \geq 1$.

we next estimate

$$
\int_{B_{s_{1} r}}[\Phi(\cdot,|D u|)]_{N}^{q-1} \Phi(x,|D u|) d x
$$

where $q>1, N \in \mathbb{N}$ and

$$
[\Phi(\cdot,|D u|)]_{N}(x):=\min \{\Phi(x,|D u(x)|), N\} .
$$

Applying Fubini's theorem, we have for sufficiently large $N$,

$$
\begin{aligned}
& \int_{B_{s_{1} r}}[\Phi(\cdot,|D u|)]_{N}^{q-1} \Phi(x,|D u|) d x \\
& =(q-1) A_{2}^{q-1} \int_{0}^{N / A_{2}} \lambda^{q-2} \int_{E\left(s_{1}, A_{2} \lambda\right)} \Phi(x,|D u|) d x d \lambda \\
& =(q-1) A_{2}^{q-1} \int_{0}^{A_{1} \lambda_{0}} \lambda^{q-2} \int_{E\left(s_{1}, A_{2} \lambda\right)} \Phi(x,|D u|) d x d \lambda \\
& \quad+(q-1) A_{2}^{q-1} \int_{A_{2} \lambda_{0}}^{N / A_{2}} \lambda^{q-2} \int_{E\left(s_{1}, A_{2} \lambda\right)} \Phi(x,|D u|) d x d \lambda=: I_{4}+I_{5} .
\end{aligned}
$$

where $A_{1}$ and $A_{2}$ are denoted in (4.3) and (4.9), respectively. Then for $I_{4}$, we have

$$
\left|I_{4}\right| \leq\left(A_{1} A_{2} \lambda_{0}\right)^{q-1} \int_{B_{s_{1} r}} \Phi(x,|D u|) d x .
$$

As for $I_{5}$, using (4.11) and Fubini's theorem, we have

$$
\begin{aligned}
\left|I_{5}\right| \leq & (q-1) A_{2}^{q-1} c_{1} \epsilon \int_{0}^{4 N} \lambda^{q-2}\left\{\int_{\left\{x \in B_{s_{2} r}: \Phi(x,|D u|)>\frac{\lambda}{4}\right\}} \Phi(x,|D u|) d x\right. \\
& \left.+\frac{1}{\delta} \int_{\left\{x \in B_{s_{2} r}: \Phi(x,|F|)+\Phi(x,|D u|)>\frac{\delta \lambda}{4}\right\}}[\Phi(x,|F|)+\Phi(x,|D \psi|)] d x\right\} d \lambda \\
\leq & (q-1)\left(4 A_{2}\right)^{q-1} c_{1} \epsilon \int_{0}^{N} \lambda^{q-2} \int_{\left\{x \in B_{s_{2} r}: \Phi(x,|D u|)>\lambda\right\}} \Phi(x,|D u|) d x d \lambda \\
& +\frac{(q-1) A_{2}^{q-1} c_{1} \epsilon}{\delta^{q}} \\
& \times \int_{0}^{\infty} \lambda^{q-2} \int_{\left\{x \in B_{s_{2} r}: \Phi(x,|F|)+\Phi(x,|D u|)>\lambda\right\}}[\Phi(x,|F|)+\Phi(x,|D \psi|)] d x d \lambda \\
\leq & \left(4 A_{2}\right)^{q-1} c_{1} \epsilon \int_{B_{s_{2} r}}[\Phi(x,|D u|)]_{N}^{q-1} \Phi(x,|D u|) d x
\end{aligned}
$$




$$
+\frac{A_{2}^{q-1} c_{1} \epsilon}{\delta^{q}} \int_{B_{s_{2} r}}[\Phi(x,|F|)+\Phi(x,|D \psi|)]^{q} d x .
$$

Therefore, choosing $\epsilon \in(0,1)$ such that $\left(4 A_{2}\right)^{q-1} c_{1} \epsilon=\frac{1}{2}$, hence $\delta$ is determined as a constant depending on $n, \nu, \Lambda, \gamma_{1}, \gamma_{2}$, and inserting the estimates for $I_{4}$ and $I_{5}$ into (4.12), we have

$$
\begin{aligned}
& \int_{B_{s_{1} r}}[\Phi(x,|D u|)]_{N}^{q-1} \Phi(x,|D u|) d x \leq \frac{1}{2} \int_{B_{s_{2} r}}[\Phi(x,|D u|)]_{N}^{q-1} \Phi(x,|D u|) d x \\
& \quad+\frac{c \lambda_{0}^{q-1}}{\left(s_{2}-s_{1}\right)^{(q-1) n}} \int_{B_{2 r}} \Phi(x,|D u|) d x+c \int_{B_{2 r}}[\Phi(x,|F|)+\Phi(x,|D \psi|)]^{q} d x .
\end{aligned}
$$

Since $1 \leq s_{1}<s_{2} \leq 2$ and the constants $c$ are independent of $s_{1}$ and $s_{2}$, in view of Lemma 2.5, we have

$$
\begin{aligned}
f_{B_{r}}[\Phi(x,|D u|)]_{N}^{q-1} \Phi(x,|D u|) d x \leq & c \lambda_{0}^{q-1} f_{B_{2 r}} \Phi(x,|D u|) d x \\
& +c \int_{B_{2 r}}[\Phi(x,|F|)+\Phi(x,|D \psi|)]^{q} d x .
\end{aligned}
$$

Applying (4.2) and using Young's inequality and Hölder's inequality, we obtain

$$
\begin{aligned}
f_{B_{r}}[\Phi(x,|D u|)]_{N}^{q-1} \Phi(x,|D u|) d x \leq & c\left(\int_{B_{2 r}} \Phi(x,|D u|) d x\right)^{q} \\
& +c f_{B_{2 r}}\left[\Phi(x,|F|)^{q}+\Phi(x,|D \psi|)^{q}+1\right] d x .
\end{aligned}
$$

Finally, by Lebesgue's monotone convergence theorem, letting $N \rightarrow \infty$, we obtain (1.12).

\section{Appendix}

We prove the existence and the uniqueness of $u \in \mathcal{K}_{\psi}^{\Phi}(\Omega)$ satisfying (1.5). For $f, g \in W^{1, \Phi}(\Omega), f=g$ (resp. $\left.f \leq q\right)$ on $\partial \Omega$ means $f-g\left(\operatorname{resp} .(f-g)_{+}\right) \in$ $W_{0}^{1, \Phi}(\Omega)$.

Theorem 5.1. Suppose that $p(\cdot)$ satisfies (1.8) for some $\delta>0$ and $R \in(0,1)$ and the nonlinearity a : $\Omega \times \mathbb{R}^{n} \rightarrow \mathbb{R}^{n}$ satisfies (2.4) and (2.9). If $\psi, g \in$ $W^{1, \Phi}(\Omega)$ with $\psi \leq g$ and $F \in L^{\Phi}\left(\Omega, \mathbb{R}^{n}\right)$, then there exists a unique $u \in \mathcal{K}_{\psi}^{\Phi}(\Omega)$ with $u=g$ on $\partial \Omega$ satisfying the variational inequality (1.5).

Proof. Note that the uniqueness directly follows from the monotonicity (2.4). Hence we prove the existence. By Poincaré's inequality for $W_{0}^{1, \Phi}(\Omega)$, see [39, Theorem 3.5], we set $\|f\|_{W_{0}^{1, \Phi}(\Omega)}:=\|D f\|_{L^{\Phi}\left(\Omega, \mathbb{R}^{n}\right)}$ in this proof, without loss of generality. We further define $\mathbf{A}: W_{0}^{1, \Phi}(\Omega) \rightarrow\left(W_{0}^{1, \Phi}(\Omega)\right)^{\prime}$, where $\left(W_{0}^{1, \Phi}(\Omega)\right)^{\prime}$ is the dual space of $W_{0}^{1, \Phi}(\Omega)$, by

$$
\mathbf{A} f(\varphi)=\int_{\Omega} \mathbf{a}(x, D f+D g) \cdot D \varphi d x
$$


and $\mathbf{F} \in\left(W_{0}^{1, \Phi}(\Omega)\right)^{\prime}$ by

$$
\mathbf{F}(\varphi)=\int_{\Omega}|F|^{p(x)-2} \log (e+|F|) F \cdot D \varphi d x .
$$

In view of Theorem 2.3 of Chapter II in [43], if for some $r>0$ there holds

$$
\mathbf{A} \varphi\left(\varphi-(\psi-g)^{+}\right)>\mathbf{F}\left(\varphi-(\psi-g)^{+}\right)
$$

for all $\varphi \in \mathcal{K}_{\psi-g}^{\Phi}(\Omega) \cap W_{0}^{1, \Phi}(\Omega)$ with $\|\varphi\|_{W_{0}^{1, \Phi}(\Omega)}>r$, then there exists $v \in$ $\mathcal{K}_{\psi-g}^{\Phi}(\Omega) \cap W_{0}^{1, \Phi}(\Omega)$ such that

$$
\mathbf{A} \varphi(\varphi-v)>\mathbf{F}(\varphi-v)
$$

for all $\varphi \in \mathcal{K}_{\psi-g}^{\Phi}(\Omega) \cap W_{0}^{1, \Phi}(\Omega)$. Therefore, $u:=v+g \in \mathcal{K}_{\psi}^{\Phi}(\Omega)$ satisfies the variational inequality (1.5).

Hence we prove (5.1). It suffices to show that

$$
\frac{\mathbf{A} \varphi\left(\varphi-(\psi-g)^{+}\right)}{\left\|\varphi-(\psi-g)^{+}\right\|_{W_{0}^{1, \Phi}(\Omega)}} \rightarrow \infty \quad \text { as } \quad\|\varphi\|_{W_{0}^{1, \Phi}(\Omega)} \rightarrow \infty .
$$

By (1.3) and (2.6) and using (2.1) and (2.2), we have

$$
\begin{gathered}
\frac{\mathbf{A} \varphi\left(\varphi-(\psi-g)^{+}\right)}{\left\|\varphi-(\psi-g)^{+}\right\|_{W_{0}^{1, \Phi}(\Omega)}} \geq \frac{\int_{\Omega} \mathbf{a}(x, D[\varphi+g]) \cdot\left(D\left[\varphi-(\psi-g)^{+}\right]\right) d x}{\|D g\|_{L^{\Phi}\left(\Omega, \mathbb{R}^{n}\right)}+\left\|D(\psi-g)^{+}\right\|_{L^{\Phi}\left(\Omega, \mathbb{R}^{n}\right)}} \\
\geq \frac{\frac{\tilde{\nu}}{2} \int_{\Omega} \Phi(x,|D[\varphi+g]|) d x-c \int_{\Omega} \Phi\left(x,\left|D\left[g-(\psi-g)^{+}\right]\right|\right) d x}{\|D \varphi\|_{L^{\Phi}\left(\Omega, \mathbb{R}^{n}\right)}+\left\|D(\psi-g)^{+}\right\|_{L^{\Phi}\left(\Omega, \mathbb{R}^{n}\right)}} .
\end{gathered}
$$

for some constant $c=c\left(n, \nu, \Lambda, \gamma_{1}, \gamma_{2}\right)>0$. Finally, using the relation (2.3) we have

$$
\begin{aligned}
& \frac{\mathbf{A} f\left(\varphi-(\psi-g)^{+}\right)}{\left\|\varphi-(\psi-g)^{+}\right\|_{W_{0}^{1, \Phi}(\Omega)}} \\
& \quad \geq \frac{\frac{\tilde{\nu}}{2}\left\{\|D \varphi\|_{L^{\Phi}\left(\Omega, \mathbb{R}^{n}\right)}^{\gamma_{1}}-\|D g\|_{L^{\Phi}\left(\Omega, \mathbb{R}^{n}\right)}^{\gamma_{1}}\right\}-c \int_{\Omega} \Phi\left(x,\left|D(\psi-g)^{+}\right|\right) d x}{\|D \varphi\|_{L^{\Phi}\left(\Omega, \mathbb{R}^{n}\right)}+\left\|D(\psi-g)^{+}\right\|_{L^{\Phi}\left(\Omega, \mathbb{R}^{n}\right)}},
\end{aligned}
$$

if $\|D g\|_{L^{\Phi}(\Omega)} \geq 1$. Consequently, since $\gamma_{1}>1$, we have (5.2).

\section{References}

[1] Acerbi, E., Fusco, N.: Partial regularity under anisotropic $(p, q)$ growth conditions. J. Differ. Equ. 107(1), 46-67 (1994)

[2] Acerbi, E., Fusco, N.: A transmission problem in the calculus of variations. Calc. Var. Partial Differ. Equ. 2(1), 1-16 (1994)

[3] Acerbi, E., Mingione, G.: Gradient estimates for the $p(x)$-Laplacean system. J. Reine Angew. Math. 584, 117-148 (2005)

[4] Acerbi, E., Mingione, G.: Gradient estimates for a class of parabolic systems. Duke Math. J. 136(2), 285-320 (2007) 
[5] Baroni, P.: Riesz potential estimates for a general class of quasilinear equations. Calc. Var. Partial Differ. Equ. 53(3-4), 803-846 (2015)

[6] Baroni, P., Colombo, M., Mingione, G.: Harnack inequalities for double phase functionals. Nonlinear Anal. 121, 206-222 (2015)

[7] Baroni, P., Colombo, M., Mingione, G.: Non-autonomous functionals, borderline cases and related function classes. St. Petersburg Math. J. 27(3), 347-379 (2016)

[8] Breit, D., Cianchi, A., Diening, L., Kuusi, T., Schwarzacher, S.: Pointwise Calderón-Zygmund gradient estimates for the $p$-Laplace system. arXiv:1510.02612

[9] Bögelein, V., Duzaar, F., Mingione, G.: Degenerate problems with irregular obstacles. J. Reine Angew. Math. 650, 107-160 (2011)

[10] Byun, S., Cho, Y., Ok, J.: Global gradient estimates for nonlinear obstacle problems with nonstandard growth. Forum Math. 28(4), 729-747 (2016)

[11] Byun, S., Cho, Y., Wang, L.: Calderón-Zygmund theory for nonlinear elliptic problems with irregular obstacles. J. Funct. Anal. 263(10), 3117-3143 (2012)

[12] Byun, S., Ok, J., Ryu, S.: Global gradient estimates for elliptic equations of $p(x)$ Laplacian type with BMO nonlinearity. J. Reine Angew. Math. 715, 1-38 (2016)

[13] Byun, S., Ok, J.: On $W^{1, q(\cdot)}$-estimates for elliptic equations of $p(x)$-Laplacian type. J. Math. Pures Appl. 106(3), 512-545 (2016)

[14] Byun, S., Wang, L.: Elliptic equations with BMO coefficients in Reifenberg domains. Commun. Pure Appl. Math. 57(10), 1283-1310 (2004)

[15] Caffarelli, L.A., Peral, I.: On $W^{1, p}$ estimates for elliptic equations in divergence form. Commun. Pure Appl. Math. 51(1), 1-21 (1998)

[16] Colombo, M., Mingione, G.: Regularity for double phase variational problems. Arch. Rat. Mech. Anal. 215(2), 443-496 (2015)

[17] Colombo, M., Mingione, G.: Bounded minimizers of double phase variational integrals. Arch. Rat. Mech. Anal. 218(1), 219-273 (2015)

[18] Colombo, M., Mingione, G.: Calderón-Zygmund estimates and non-uniformly elliptic operators. J. Funct. Anal. 270(4), 1416-1478 (2016)

[19] Cupini, G., Leonetti, F., Mascolo, E.: Existence of weak solutions for elliptic systems with $p, q$-growth. Ann. Acad. Sci. Fenn. Math. 40(2), 645-658 (2015)

[20] DiBenedetto, E., Manfredi, J.: On the higher integrability of the gradient of weak solutions of certain degenerate elliptic systems. Am. J. Math. 115(5), 11071134 (1993)

[21] Diening, L., Harjulehto, P., Hästö, P., Ružička, M.: Lebesgue and sobolev spaces with variable exponents. In: Lecture Notes in Mathematics, vol. 2017. Springer, Berlin (2011) 
[22] Diening, L., Schwarzacher, S.: Global gradient estimates for the $p(\cdot)$ Laplacian. Nonlinear Anal. 106, 70-85 (2014)

[23] Eleuteri, M., Habermann, J.: Calderón-Zygmund type estimates for a class of obstacle problems with $p(x)$ growth. J. Math. Anal. Appl. 372(1), 140-161 (2010)

[24] Erhardt, A.: Calderón-Zygmund theory for parabolic obstacle problems with nonstandard growth. Adv. Nonlinear Anal. 3(1), 15-44 (2014)

[25] Fusco, N., Sbordone, C.: Higher integrability of the gradient of minimizers of functionals with nonstandard growth conditions. Commun. Pure Appl. Math. 43(5), 673-683 (1990)

[26] Fusco, N., Sbordone, C.: Some remarks on the regularity of minima of anisotropic integrals. Commun. Partial Differ. Equ. 18(1-2), 153-167 (1993)

[27] Giannetti, F., Passarellidi Napoli, A.: Regularity results for a new class of functionals with nonstandard growth conditions. J. Differ. Equ. 254(3), 12801305 (2013)

[28] Han, Q., Lin, F.: Elliptic Partial Differential Equation. Courant Institute of Math. Sci., New York University (1997)

[29] Harjulehto, P., Hästö, P., Klén, R.: Generalized Orlicz Spaces and Related PDE. Nonlinear Anal. 143, 155-173 (2016)

[30] Hästö, P.: Counter-examples of regularity in variable exponent Sobolev spaces. In: The $p$-Harmonic Equation and Recent Advances in Analysis (Manhattan, KS, 2004). Contemp. Math., vol. 367, pp. 133-143. Amer. Math. Soc., Providence (2005)

[31] Iwaniec, T.: Projections onto gradient fields and $L^{p}$-estimates for degenerated elliptic operators. Studia Math. 75(3), 293-312 (1983)

[32] Kuusi, T., Mingione, G.: Linear potentials in nonlinear potential theory. Arch. Rat. Mech. Anal. 207, 215-246 (2013)

[33] Leonetti, F.: Higher integrability for minimizers of integral functionals with nonstandard growth. J. Differ. Equ. 112(2), 308-324 (1994)

[34] Leonetti, F., Mascolo, E.: Local boundedness for vector valued minimizers of anisotropic functionals. Z. Anal. Anwend. 31(3), 357-378 (2012)

[35] Lieberman, G.M.: The natural generalization of the natural conditions of Ladyzenskaja and Uralâtzeva for elliptic equations. Commun. Partial Differ. Equ. 16(2-3), 311-361 (1991)

[36] Marcellini, P.: Regularity of minimizers of integrals of the calculus of variations with non standard growth conditions. Arch. Rat. Mech. Anal. 105, 267$284(1989)$

[37] Marcellini, P.: Regularity and existence of solutions of elliptic equations with $p, q$-growth conditions. J. Differ. Equ. 90, 1-30 (1991) 
[38] Mingione, G.: The Calderón-Zygmund theory for elliptic problems with measure data. Ann. Sc. Norm. Super. Pisa Cl. Sci.(5) 6(2), 195-261 (2007)

[39] Ok, J.: Gradient estimates for elliptic equations with $L^{p(\cdot)} \log L$ growth. Calc. Var. Partial Differ. Equ. 55(2), 55-26 (2016)

[40] Ok, J.: Harnack inequality for a class of functionals with non-standard growth via De Giorgi's method. Adv. Nonlinear Anal. doi:10.1515/anona-2016-0083

[41] Ok, J.: Regularity results for a class of obstacle problems with nonstandard growth (preprint)

[42] Scheven, C.: Existence of localizable solutions to nonlinear parabolic problems with irregular obstacles. Manuscripta Math. 146(1-2), 7-63 (2015)

[43] Showalter, R.E.: Monotone operators in banach space and nonlinear partial differential equations. In: Mathematical Surveys and Monographs, vol. 49. American Mathematical Society, Providence (1997)

[44] Zhikov, V.V.: On Lavrentiev's phenomenon. Russian J. Math. Phys. 3(2), 249269 (1995)

Jihoon Ok

School of Mathematics

Korea Institute for Advanced Study

Seoul 02455

Korea

e-mail: jihoonok@kias.re.kr

Received: 15 June 2016.

Accepted: 29 July 2016. 